\title{
Prognostic Value of Noninvasive Fibrosis Scores in Primary Biliary Cholangitis
}

\author{
Yundong Qu1, Lei Wang1, Qian Ye1, Yuan Zhang2, Juan Xiao² and Tao Li1 \\ IDepartment of Infectious Diseases and Hepatology, The Second Hospital of Shandong University, Jinan 250033, China \\ ${ }^{2}$ Center of Evidence-based Medicine, Institute of Medical Sciences, The Second Hospital of Shandong University, Jinan 250033, China
}

\begin{abstract}
Objective: To explore the prognostic value of several widely used noninvasive fibrosis scores (NIFS) for the mortality due to liver-related events in Chinese primary biliary cholangitis (PBC) population.

Study Design: An observational study.

Place and Duration of Study: Department of Infectious Diseases and Hepatology, the Second Hospital of Shandong University, Jinan, China, from August 2008 to July 2018.

Methodology: Patients were diagnosed as PBC when they fulfilled at least two of the following criteria: presence of antimitochondrial antibodies (AMA), or other PBC-specific autoantibodies; and/or biochemical evidence of cholestasis; and/or histological evidence of liver biopsy. Patients were excluded if they were just started UDCA administration within last year, followed up for less than a year, diagnosed as overlap syndrome, or suffered from other coexisting hepatic diseases. Clinical data were recorded and scores of 11 generally accepted NIFS were calculated. Cox proportional hazards model was performed to explore independent predictors of liver-related mortality.

Results: Sixty-five PBC patients were included in the current cohort. Five patients died due to liver-related events during a median of 35-month follow-up. The 5-year cumulative survival rate was $88.4 \%$. Non-survival patients were characterised with lower platelet count $(p=0.049)$, lower level of albumin $(p=0.018)$, higher fibrosis index $(p<0.001)$ and higher Doha score $(p=0.006)$. Multivariate Cox regression analysis identified fibrosis index (HR 17.449, $95 \% \mathrm{Cl} 1.410-215.989$, $p=0.026)$ and Doha score (HR 1.782, $95 \% \mathrm{Cl} 1.146-2.771, p=0.010)$ as independent predictors for liver-related mortality of PBC patients.

Conclusion: Fibrosis index and Doha score could serve as valuable prognostic factors for liver-related mortality in Chinese PBC population.
\end{abstract}

Key Words: Primary biliary cholangitis, Noninvasive fibrosis scores, Mortality.

How to cite this article: Qu Y, Wang L, Ye Q, Zhang Y, Xiao J, Li T. Prognostic value of noninvasive fibrosis scores in primary biliary cholangitis. J Coll Physicians Surg Pak 2019; 29(11):1101-5.

\section{INTRODUCTION}

Primary biliary cholangitis (PBC) is a chronic autoimmune liver disease and cholestasis is confirmed as a feature of disease course.1,2 The etiology of PBC is still unknown, genetic predisposition and environmental factors have been indicated as important pathogenic factors. 3 Outcomes in PBC generally rested on the development of cirrhosis and its complications.1,2

Ursodeoxycholic acid (UDCA) is the first line drug approved for the treatment of patients with PBC, which has been proven to ameliorate biochemical indices, delay histologic progression and improve long term survival.1,4 However, about one-third of PBC patients may not obtain perfect biochemical response after 12 months' therapy with UDCA and there may be factors

Correspondence to: Tao Li, Department of Infectious Diseases and Hepatology, the Second Hospital of Shandong University,

247 Beiyuan Road, Jinan, Shandong 250033, China

E-mail:022303052@163.com

Received: November 19, 2018; Revised: April 13, 2019;

Accepted: May 25, 2019 independently associated with disease progression and death due to liver-related events. 4,5 Several models have been designed to evaluate biochemical response to UDCA, including the Rotterdam criteria, ${ }^{6}$ Barcelona criteria, ${ }^{7}$ Paris I criteria, ${ }^{8}$ Paris II criteria, ${ }^{5}$ and Toronto criteria. ${ }^{9}$ These models are based on the improvement of several biochemical markers after 6-12 months of UDCA treatment, such as alkaline phosphatase (ALP), gammaglutamyl transpeptidase (GGT), albumin and total bilirubin (TB). Recently, two new models (the GLOBE score and the UK-PBC score), 10,11 have been introduced by including some other factors, such as age and platelet count, to predict long-term outcomes of PBC. However, as the above predictive models including indices of the baseline and 1 year after UDCA therapy, it is difficult to judge the prognosis of $\mathrm{PBC}$ patients prior to initiating therapy.

Several noninvasive fibrosis scores (NIFS), which could be calculated from liver function tests and other routine blood tests, such as aspartate aminotransferas (AST)platelet ratio index (APRI) and Lok score, have been widely used for determination of liver fibrosis. These noninvasive score systems have also been shown a 
good prognostic value for prognosis of patients with chronic hepatitis $\mathrm{C}(\mathrm{CHC}) .{ }^{12}$ However, only a few studies in literature have evaluated prognostic value of NIFS for the long-term survival of PBC population.

The present study was performed to explore prognostic value of several widely used NIFS for the mortality due to liver-related events in Chinese PBC population.

\section{METHODOLOGY}

It was observational study where all patients diagnosed with PBC and treated at the Second Hospital of Shandong University were recruited between August 2008 and July 2018. The diagnosis of PBC should fulfill at least two of the following criteria: ${ }^{1}$ (1) presence of anti-mitochondrial antibodies (AMA), or other PBC-specific autoantibodies, including anti-sp100 or anti-glycoprotein 210 (antigp210); (2) biochemical evidence of cholestasis (elevated ALP levels); and (3) histological evidence of liver biopsy, such as non-suppurative destructive cholangitis and destruction of interlobular bile ducts. Patients were excluded if they were: just started UDCA administration within last year, followed up for less than one year, diagnosed as overlap syndrome, suffered from other coexisting hepatic diseases including chronic hepatitis $\mathrm{B}$ $(\mathrm{CHB})$ and $\mathrm{CHC}$ infection, alcoholic liver disease, steatohepatitis, and hereditary metabolic liver disease.

Patients' age, gender, platelet count, liver biochemistry, creatinine, and international normalised ratio (INR) were recorded. Scores of 11 NIFS deriving from blood tests for pre-treatment assessment of liver fibrosis were calculated, including fibrosis-4 index, Lok score, Fibroalpha, GUCl score, AST-ALT ratio (AAR), Fibrosis index, Fibro-Q score, Doha score, Age platelet count index, King's score and AST-platelet count ratio index (APRI) (Table I). ${ }^{12,13}$

The endpoint event was set as death due to liver-related events, such as hepatocellular carcinoma (HCC), hepatic encephalopathy, spontaneous bacterial peritonitis, hepatorenal syndrome and gastrointestinal bleeding. The protocol of the current study followed guidelines of the Helsinki Declaration and has been approved by the Ethical Committee of the Second Hospital of Shandong University.

All data were analysed using IBM SPSS Statistics Version 22.0 (IBM Corp., Armonk, NY, USA). KolmogorovSmirnov test was performed to calculate the normality of continuous variables. Data were expressed by using mean \pm standard deviation (SD) or median [interquartile range (IQR)] for continuous variables. Student t-test or Mann-Whitney test were selected for the comparisons of continuous statistics. Cox proportional hazards model was performed to explore independent predictors of liver-related mortality. Covariates brought into multivariable analysis included: age at diagnosis of $\mathrm{PBC}$, gender, alanine aminotransferase (ALT), aspartate aminotransferas (AST), GGT, TB, albumin, platelet count, INR, creatinine, and a specific NIFS. If some covariates were included in the given NIFS, they were removed from the multivariable analysis. A p-value less than 0.05 was considered as statistically significant.

Table I: Calculations of included noninvasive fibrosis scores (NIFS). ${ }^{12,20}$

\begin{tabular}{|c|c|}
\hline NIFS & Calculations \\
\hline Fibrosis-4 index & $($ Age $\times \mathrm{AST}) /(\mathrm{PLT} \times \mathrm{ALT}(1 / 2))$ \\
\hline Lok score & $\begin{array}{l}\text { Log odds }=-5.56-0.0089 \times \text { platelet count }+1.26 \times \\
(\text { AST/ALT) }+5.27 \times \text { INR; Predicted probability }=[\exp \\
(\text { log odds })] /[1+\exp (\text { log odds })]\end{array}$ \\
\hline Fibro-alpha & $\begin{array}{l}1.35+[\mathrm{AFP} \times 0.009584]+[(\mathrm{AST}) /(\mathrm{ALT}) \times 0.243]- \\
(\mathrm{PLT} \times 0.001624)\end{array}$ \\
\hline GUCl score & Normalized AST $\times$ INR $\times 100 /$ PLT \\
\hline AST-ALT ratio (AAR) & AST/ALT \\
\hline Fibrosis index & $8.0-(0.01 \times \mathrm{PLT})-$ serum albumin \\
\hline Fibro-Q score & $($ Age $\times$ AST $\times$ INR $) /($ ALT $\times \mathrm{PLT})$ \\
\hline Doha score & $8.5-0.2 \times$ albumin $+0.01 \times$ AST $-0.02 \times \mathrm{PLT}$ \\
\hline Age platelet count index & Age/PLT \\
\hline King's score & $($ Age $\times$ AST $\times$ INR $) / P L T$ \\
\hline $\begin{array}{l}\text { AST-platelet count ratio } \\
\text { index (APRI) }\end{array}$ & $($ Normalized AST/PLT $) \times 100$ \\
\hline
\end{tabular}

NIFS, Noninvasive fibrosis scores; ALT, alanine aminotransferase; AST, aspartate aminotransferas; GGT, gamma-glutamyl transferase; INR, international normalised ratio; PLT, platelet count.

Normalised $A S T=$ observed AST/upper limit of normal AST

Age was expressed as years; ALT and AST were expressed as U/L; PLT was expressed as $109 / \mathrm{L}$; AFP was expressed as IU/ml; albumin was expressed as $\mathrm{g} / \mathrm{dl}$.

Table II: Characteristics of included patients.

\begin{tabular}{|c|c|c|c|c|}
\hline & Total $(n=65)$ & Alive $(n=60)$ & Death $(n=5)$ & $\mathrm{p}$-value \\
\hline Age (years) & $61.1 \pm 10.9$ & $61.0 \pm 10.6$ & $63.2 \pm 15.0$ & 0.662 \\
\hline $\begin{array}{l}\text { Gender } \\
\text { (male / female) }\end{array}$ & $7 / 58$ & $6 / 54$ & $1 / 4$ & 0.488 \\
\hline $\operatorname{ALT}(U / L)^{*}$ & $72.0(40.0-102.0)$ & $71.0(38.5-102.5)$ & $72.0(57-364)$ & 0.510 \\
\hline AST $(U / L)^{*}$ & $70.0(53.0-97.0)$ & $69.5(49.3-96.5)$ & 73 (63.5-398.5) & 0.272 \\
\hline$\overline{\mathrm{GGT}}(\mathrm{U} / \mathrm{L})^{*}$ & $\begin{array}{c}224.0 \\
(127.5-381.0)\end{array}$ & $\begin{array}{c}217.5 \\
(123.8-390.5)\end{array}$ & $\begin{array}{c}275 \\
(186-383.5)\end{array}$ & 0.747 \\
\hline $\begin{array}{l}\text { Bilirubin } \\
\text { (umol/L)* }^{*}\end{array}$ & $22.0(14.7-37.7)$ & $21.9(13.6-38.7)$ & $30.5(17.0-43.9)$ & 0.464 \\
\hline $\begin{array}{l}\text { Platelet count } \\
(109 / \mathrm{L})\end{array}$ & $158.8 \pm 63.1$ & $163.3 \pm 63.0$ & $105.6 \pm 36.5$ & 0.049 \\
\hline $\mathrm{INR}^{*}$ & $0.96(0.93-1.00)$ & $0.96(0.93-1.00)$ & $0.92(0.88-0.99)$ & 0.203 \\
\hline Albumin (g/L) & $34.8 \pm 6.5$ & $35.4 \pm 6.4$ & $28.3 \pm 3.6$ & 0.018 \\
\hline $\begin{array}{l}\text { Creatinine } \\
(\text { umol/L) }\end{array}$ & $53.6(47.2-60.2)$ & $53.6(46.9-60.2)$ & $51.3(47.6-208.6)$ & 0.858 \\
\hline $\begin{array}{l}\text { Fibrosis-4 } \\
\text { index }\end{array}$ & $3.46(2.22-6.00)$ & $3.29(2.19-5.69)$ & $6.28(4.31-21.7)$ & 0.062 \\
\hline Lok score & $0.43 \pm 0.24$ & $0.43 \pm 0.25$ & $0.48 \pm 0.12$ & 0.657 \\
\hline Fibro-alpha* $^{*}$ & $1.42(1.29-1.51)$ & $1.41(1.28-1.51)$ & $1.50(1.43-1.60)$ & 0.154 \\
\hline GUCI score* & $1.04(0.63-1.95)$ & $1.01(0.61-1.82)$ & $1.62(1.07-14.45)$ & 0.103 \\
\hline $\begin{array}{l}\text { AST-ALT ratio } \\
(\text { AAR })^{*}\end{array}$ & $1.03(0.82-1.48)$ & $1.03(0.79-1.48)$ & $1.08(0.99-1.47)$ & 0.510 \\
\hline Fibrosis index & $2.92 \pm 1.05$ & $2.83 \pm 1.04$ & $4.11 \pm 0.26$ & $<0.001$ \\
\hline Fibro-Q score* & $0.44(0.27-0.68)$ & $0.41(0.26-0.65)$ & $0.67(0.45-1.11)$ & 0.114 \\
\hline Doha score & $5.68 \pm 1.86$ & $5.50 \pm 1.65$ & $7.82 \pm 2.97$ & 0.006 \\
\hline $\begin{array}{l}\text { Age platelet } \\
\text { count index }\end{array}$ & $0.37(0.29-0.59)$ & $0.37(0.28-0.57)$ & $0.66(0.36-1.11)$ & 0.092 \\
\hline King's score* & $26.2(16.4-48.8)$ & $26.1(16.1-47.6)$ & $48.5(31.6-488.3)$ & 0.120 \\
\hline $\begin{array}{l}\text { AST-platelet } \\
\text { count ratio } \\
\text { index (APRI)* }\end{array}$ & $1.10(0.65-1.91)$ & $0.98(0.64-1.83)$ & $1.67(1.17-14.68)$ & 0.074 \\
\hline
\end{tabular}

$\overline{A L T}$, alanine aminotransferase; AST, aspartate aminotransferas; GGT, gamma-glutamyl transferase; INR, international normalized ratio; IQR, interquartile range.

*Median (interquartile range). 
Table III: Univariate and multivariate cox regression analysis revealing predictive value of 11 selected NIFS.

\begin{tabular}{|c|c|c|c|c|c|c|}
\hline & \multicolumn{3}{|c|}{ Univariate analysis } & \multicolumn{3}{|c|}{ Multivariate analysis } \\
\hline & $\mathrm{B}$ & $\operatorname{HR}(95 \% \mathrm{Cl})$ & $p$-value & $\mathrm{B}$ & $\mathrm{HR}(95 \% \mathrm{Cl})$ & $p$-value \\
\hline Fibrosis-4 index & 0.110 & $1.116(1.028-1.212)$ & 0.009 & 1.320 & $3.744(0.146-95.759)$ & 0.425 \\
\hline Lok score & 0.767 & $2.154(0.064-72.126)$ & 0.668 & & & \\
\hline Fibro-alpha & 1.269 & $3.559(0.095-132.658)$ & 0.492 & & & \\
\hline GUCI score & 0.134 & $1.143(1.026-1.273)$ & 0.015 & 2.414 & $11.181(0.015-8183.793)$ & 0.473 \\
\hline AST-ALT ratio (AAR) & -0.127 & $0.880(0.256-3.027)$ & 0.840 & & & \\
\hline Fibrosis index & 2.712 & $15.056(2.000-113.317)$ & 0.008 & 2.859 & $17.449(1.410-215.989)$ & 0.026 \\
\hline Fibro-Q score & 0.626 & $1.871(0.582-6.018)$ & 0.293 & & & \\
\hline Doha score & 0.467 & $1.594(1.120-2.271)$ & 0.010 & 0.578 & $1.782(1.146-2.771)$ & 0.010 \\
\hline Age platelet count index & 2.832 & $16.979(1.395-206.673)$ & 0.026 & Not in the equation & & \\
\hline King's score & 0.004 & $1.004(1.001-1.007)$ & 0.008 & 0.050 & $1.052(0.966-1.145)$ & 0.246 \\
\hline AST-platelet count ratio index (APRI) & 0.133 & $1.143(1.027-1.272)$ & 0.015 & 1.292 & $3.639(0.211-62.873)$ & 0.374 \\
\hline
\end{tabular}

NIFS: Noninvasive fibrosis scores; ALT: Alanine aminotransferase; AST: Aspartate aminotransferas.

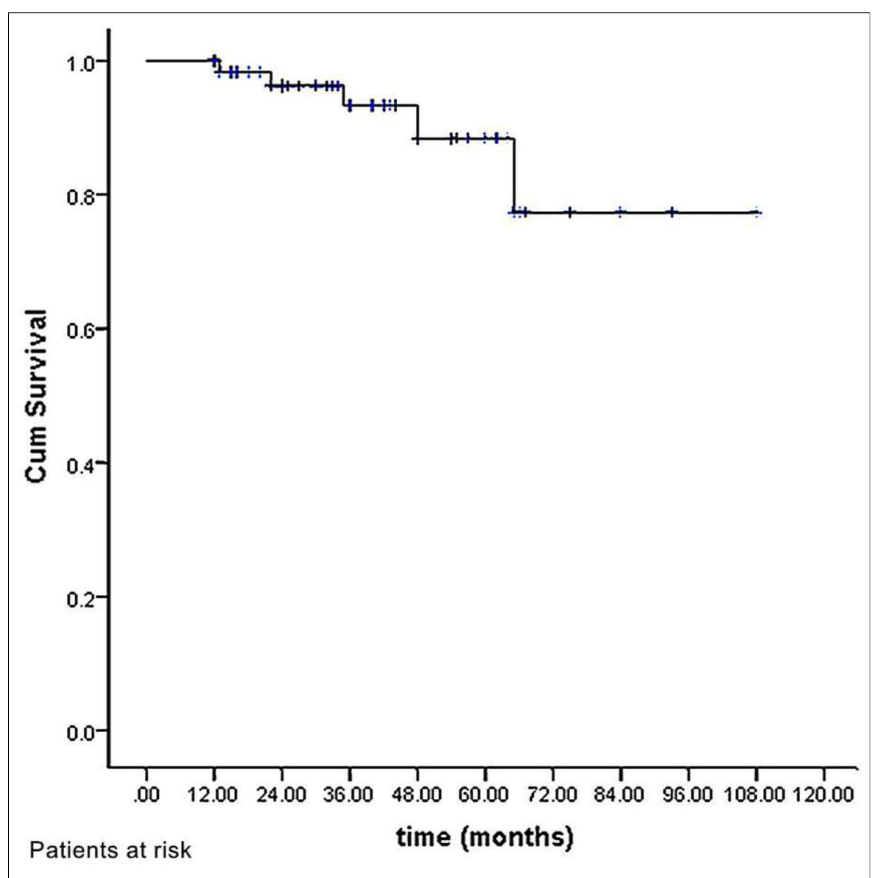

Figure 1: Cumulative survival curve of included primary biliary cholangitis patients.

\section{RESULTS}

Eighty-four patients were preliminarily identified and 19 patients were not recruited in the present cohort because of exclusion criteria. Hence, the present study included 65 PBC patients. Twelve patients (18.5\%) were diagnosed as PBC on liver biopsy. Most of the included patients were females (58 patients, $89.2 \%$ ) and the mean age at diagnosis of PBC was 61.1 years. Data of ultrasound, computer tomography (CT) or MRI were available for all included patients. Typical imaging findings of liver cirrhosis were observed in 39 patients, of whom 11 patients, 13 patients and 15 patients were evaluated as Child-Pugh Class A, B and C, respectively. Other 26 patients were diagnosed as chronic liver diseases or no obvious abnormality by ultrasound, CT or MRI. HCC occurred in two patients (3.1\%) after 12 and 48-month follow-up, respectively. During a median of 35-month (IQR 19 to 51 months) follow-up, five patients
$(7.7 \%)$ died because of liver-related events and the 5 -year cumulative survival rate was $88.4 \%$ (Figure I). Non-survival patients were characterised with lower platelet count $(p=0.049)$ and lower level of albumin $(p=0.018$, Table II).

The predictive value of 11 generally accepted NIFS were evaluated $($ Table I). Fibrosis index $(p<0.001)$ and Doha score $(p=0.006)$ were significantly higher in non-survival PBC patients (Table II). Univariate analysis revealed that higher Fibrosis-4 index $(p=0.009), \mathrm{GUCl}$ score $(p=0.015)$, Fibrosis index $(p=0.008)$, Doha score $(p=0.010)$, Age platelet count index $(p=0.026)$, King's score $(p=0.008)$ and APRI $(p=0.015)$ may be related to worse clinical prognosis (death because of liver-related events). However, in multivariate Cox regression analysis, only Fibrosis index (HR $17.449,95 \% \mathrm{Cl} 1.410-$ $215.989, p=0.026)$ and Doha score $(\mathrm{HR} 1.782,95 \% \mathrm{Cl}$ 1.146-2.771, $p=0.010$ ) were identified as independent predictors for liver-related mortality of PBC population (Table III ).

\section{DISCUSSION}

Although several models including baseline characteristics and biochemical response to UDCA have been approved to predict long-term outcomes of PBC, the prognostic value of parameters prior to initiating therapy is still unknown. Cirrhosis has been identified as an independent predictor for transplant-free survival in Chinese PBC patients; 3,14 however, the diagnosis of cirrhosis made by imaging features or findings of portal hypertension (including ascites, splenomegaly and varices) may be delayed and have low sensitivity. NIFS are routinely available in clinical practice and have been widely used for the evaluation of liver fibrosis and cirrhosis.12,13 Therefore, the evaluation of NIFS prior to UDCA therapy for long-term survival of PBC patients is promising and should be of great clinical significance.

The current study attempts to explore the prognostic value of several widely used NIFS for the mortality due to liver-related events in Chinese PBC population. Based on a long-term observational cohort recruiting 65 PBC patients, the present research selected 11 NIFS 
arriving from liver function tests and other routine blood tests to identify Fibrosis index and Doha score as valuable prognostic factors.

Parameters contained in both Fibrosis index and Doha score included serum albumin and platelet count. Albumin could reflect the synthetic ability of hepatocytes. Lower levels of albumin at the time of diagnosis have been proven to identify PBC patients with a worse prognosis.4,10,11,15 A 10-year observational UDCAtreated $\mathrm{PBC}$ cohort also revealed that the long-term survival of PBC population with normal levels of bilirubin and albumin could be comparable to that of general population. ${ }^{16}$ The above findings emphasised the prognostic value of albumin. Platelet counts could reflect the level of portal hypertension and has been considered as a serum marker of liver fibrosis and cirrhosis. Previous studies also revealed the prognostic value of platelet count for adverse events.2,10,11 The baseline levels of albumin and platelet count were also evaluated in two recently developed scoring systems for outcomes of PBC (the GLOBE, 10 and UK-PBC systems), ${ }^{11}$ revealing the validated prognostic value of the two parameters.

Trivedi et al. concluded that higher APRI is associated with risk of liver transplant (LT) or death. ${ }^{2}$ In this study, the prognostic value of APRI was also significant in univariate analysis $(p=0.015)$; however, in multivariate Cox regression analysis, ARPI was not related to the mortality due to liver-related events. The difference might be because of the interaction of different parameters (e.g. AST, bilirubin, albumin and platelet count). Discrepancy of PBC patients between Western and Asian countries should also be considered. Further validation with larger cohort is needed in the future.

Yang et al. reported that anti-gp210 could serve as a predictive indicator for the prognosis of Chinese PBC population and should be an important supplement to the GLOBE score and UK-PBC score. 15 As positive anti-gp210 in PBC patients is correlated with more severe cholestatic manifestations and higher risk of liver failure, 15,17 the combined prognostic value of antigp210 and Fibrosis index or Doha score should be investigated. However, only a small proportion of included PBC patients in our cohort were examined with anti-gp210 and this parameter was not brought into the current statistical analysis.

In the present cohort, 5 patients died because of liverrelated events during a median of 35-month follow-up. The 5 -year cumulative survival rate was $88.4 \%$, which was consistent with previous studies performed in Asian populations. 14,18 The incidences of HCC were as low as $0.76 \%-5.9 \%$ in PBC patients, 19 and were mainly found in patients with advanced stage of the disease.17 In the current study, HCC occurred in 2 patients (3.1\%) after 12 and 48 months' follow-up, respectively, which was also similar to previous studies. $14,18,19$ Both of HCC patients were diagnosed in cirrhotic status before the start of
UDCA. The present study did not explore the risk factors for HCC because of the limited number of carcinoma patients.

There are several limitations of the present study. First, the sample size was relatively small. However, the results were valid, which were consistent with the performance of various prognostic models. Second, the relatively short follow-up time (range 12 to 108 months) may restrict some prognostic models in predicting clinical outcomes after long-term follow-up, which may omit some valuable NIFS.

\section{CONCLUSION}

Two valuable NIFS (Fibrosis index and Doha score) arriving from routine pretreatment laboratory parameters were identified for the evaluation of liver-related mortality in Chinese PBC population. These predictive NIFS can be applied easily before treatment of PBC and may be helpful in the evaluation of long-term prognosis.

\section{CONFLICT OF INTEREST:}

Authors declared no conflict of interest.

\section{AUTHORS' CONTRIBUTION:}

YQ: Conception of the manuscript, study design, patients' follow-up, data collection, statistical analysis, manuscript writing and revision.

LW: Patients' follow-up and manuscript revision.

QY: Patients' follow-up and data collection.

YZ: Study design, statistical analysis, and manuscript revision.

JX: Study design, statistical analysis.

TL: Conception of the manuscript, study design, patients' follow-up, data collection, statistical analysis, manuscript writing and revision.

\section{REFERENCES}

1. Lindor KD, Bowlus CL, Boyer J, Levy C, Mayo M. Biliary cholangitis: 2018 practice guidance from the American association for the study of liver diseases. Hepatology 2019; 69:394-419

2. Trivedi PJ, Bruns T, Cheung A, Li KK, Kittler C, Kumagi T, et al. Optimising risk stratification in primary biliary cirrhosis: AST/platelet ratio index predicts outcome independent of ursodeoxycholic acid response. J Hepatol 2014; 60:1249-58.

3. Gatselis NK, Zachou K, Lygoura V, Azariadis K, Arvaniti P, Spyrou E, et al. Geoepidemiology, clinical manifestations and outcome of primary biliary cholangitis in Greece. Eur J Intern Med 2017; 42:81-8.

4. Invernizzi $P$, Floreani $A$, Carbone $M$, Marzioni $M$, Craxi $A$, Muratori L, et al. Primary biliary cholangitis: Advances in management and treatment of the disease. Dig Liver Dis 2017; 49:841-6.

5. Corpechot $\mathrm{C}$, Chazouilleres $\mathrm{O}$, Poupon $\mathrm{R}$. Early primary biliary cirrhosis: Biochemical response to treatment and prediction of long-term outcome. J Hepatol 2011; 55:1361-7. 
6. Kuiper EM, Hansen BE, de Vries RA, den Ouden-Muller JW, van Ditzhuijsen TJ, Haagsma EB, et al. Improved prognosis of patients with primary biliary cirrhosis that have a biochemical response to ursodeoxycholic acid. Gastroenterology 2009; 136:1281-7

7. Pares A, Caballeria L, Rodes J. Excellent long-term survival in patients with primary biliary cirrhosis and biochemical response to ursodeoxycholic acid. Gastroenterology 2006; 130:715-20.

8. Corpechot $\mathrm{C}$, Abenavoli L, Rabahi N, Chrétien $\mathrm{Y}$, Andréani T, Johanet $\mathrm{C}$, et al. Biochemical response to ursodeoxycholic acid and long-term prognosis in primary biliary cirrhosis. Hepatology 2008; 48:871-7.

9. Kumagi T, Guindi M, Fischer SE, Arenovich T, Abdalian R, Coltescu $\mathrm{C}$, et al. Baseline ductopenia and treatment response predict long-term histological progression in primary biliary cirrhosis. Am J Gastroenterol 2010; 105:2186-94.

10. Lammers WJ, Hirschfield GM, Corpechot C, Nevens F, Lindor KD, Janssen $\mathrm{HL}$, et al. Development and validation of a scoring system to predict outcomes of patients with primary biliary cirrhosis receiving ursodeoxycholic acid therapy. Gastroenterology 2015; 149:1804-12.e4.

11. Carbone M, Sharp SJ, Flack S, Paximadas D, Spiess K, Adgey C, et al. The UK-PBC risk scores: Derivation and validation of a scoring system for long-term prediction of end-stage liver disease in primary biliary cholangitis. Hepatology 2016; 63: 930-50.

12. Thandassery RB, Kaabi SA, Soofi ME, Tharian B, Singh R. Noninvasive serum models to predict significant liver related events in chronic hepatitis C. Hepatol Int 2017; 11:401-8.
13. Thandassery RB, Al Kaabi S, Soofi ME, Mohiuddin SA, John AK, Al Mohannadi $\mathrm{M}$, et al. Mean platelet volume, red cell distribution width to platelet count ratio, globulin platelet index, and 16 other indirect noninvasive fibrosis scores: How much do routine blood tests tell about liver fibrosis in chronic hepatitis C? J Clin Gastroenterol 2016; 50:518-23.

14. Cheung KS, Seto WK, Fung J, Lai CL, Yuen MF. Epidemiology and natural history of primary biliary cholangitis in the Chinese: A territory-based study in Hong Kong between 2000 and 2015. Clin Transl Gastroenterol 2017; 8:e116.

15. Yang F, Yang Y, Wang Q, Wang Z, Miao Q, Xiao X, et al. The risk predictive values of UK-PBC and GLOBE scoring system in Chinese patients with primary biliary cholangitis: The additional effect of anti-gp210. Aliment Pharmacol Ther 2017; 45:733-43.

16. ter Borg PC, Schalm SW, Hansen BE, van Buuren HR. Prognosis of ursodeoxycholic Acid-treated patients with primary biliary cirrhosis. Results of a 10-year cohort study involving 297 patients. Am J Gastroenterol 2006; 101:2044-50.

17. Lammers WJ, Kowdley KV, van Buuren HR. Predicting outcome in primary biliary cirrhosis. Ann Hepatol 2014; 13:316-26.

18. Kim KA, Ki M, Choi HY, Kim BH, Jang ES, Jeong SH. Populationbased epidemiology of primary biliary cirrhosis in South Korea. Aliment Pharmacol Ther 2016; 43:154-62.

19. Harada K, Hirohara J, Ueno Y, Nakano T, Kakuda Y, Tsubouchi H, et al. Incidence of and risk factors for hepatocellular carcinoma in primary biliary cirrhosis: National data from Japan. Hepatology 2013; 57:1942-9. 